\title{
Calcific Metamorphosis \\ - A review of literature and clinical management
}

SADJ July 2020, Vol. 75 No. 6 p316 - p322

PJ van der Vyver ${ }^{1}$, M Vorster ${ }^{2}$, CH Jonker ${ }^{3}$, N Potgieter ${ }^{4}$

\section{INTRODUCTION}

Calcific metamorphosis (CM) or pulp canal obliteration (Fig. 1A-C) is a common occurrence following concussion and subluxation injuries. ${ }^{1,2}$ Although the exact mechanism by which the canal obliterates is unknown, it is believed to be related to neurovascular damage and deposition of hard tissue within the canal. ${ }^{3,4}$

This calcification of the pulp canal space results in a loss of translucency leaving the crown with a yellow discolouration (Fig. 2 and 3). ${ }^{5} \mathrm{CM}$ can be clinically detected as early as three months after injury but remains undetected in most cases for up to a year after trauma. ${ }^{6-8}$

Asymptomatic teeth presenting with $\mathrm{CM}$ do not initially require treatment other than annual review. ${ }^{1,9}$ However, the pulp status within partially obliterated canals may eventually lead to apical pathology requiring treatment.10-14

Treatment protocols and exact intervention times for the treatment of CM remains controversial and a challenge for even the skilled clinician. ${ }^{15-17}$

\section{Aetiology and Incidence}

$\mathrm{CM}$ is a common outcome of trauma frequently associated with anterior teeth of young adults and is characterised by partial or total obliteration of the root canal. ${ }^{6,8,15}$

Author affiliations:

1. Peet J van der Vyver: BChD, PG Dip Dent (Endo), PG Dip Dent (Aesthet Dent), MSc, PhD (Pret), Department of Odontology, School of Dentistry, University of Pretoria, Pretoria, South Africa. ORCID Number: 0000-0003-1951-6042

2. Martin Vorster: BChD, PG Dip Dent (Endo), MSc (Pret), Department of Odontology, School of Dentistry, University of Pretoria, Pretoria, South Africa. ORCID Number: 0000-0003-4470-1530

3. Casper H Jonker: BChD (Pret), Dip Odont (Endo), MSc (Pret), Department of Operative Dentistry, Sefako Magatho Health Sciences University, Ga-Rankuwa, South Africa. ORCID Number: 0000-0002-9110-5208

4. Nicoline Potgieter: BChD, PG Dip Dent (Endo), MSc (Pret), Department of Odontology, School of Dentistry, University of Pretoria, Pretoria, South Africa. ORCID Number: 0000-0003-4061-3322

Corresponding author: Martin Vorster

Department of Odontology, School of Dentistry, University of Pretoria, Gauteng, South Africa.

Email: martin.vorster@up.co.za

Author contributions:

1. Peet $\mathbf{J}$ van der Vyver: Treated patients, followed-up with patients, scientific writing and proofreading of the manuscript - $25 \%$

2. Martin Vorster: Scientific writing and proofreading of the manuscript - $25 \%$

3. Casper $\mathrm{H}$ Jonker: Scientific writing and proofreading of the manuscript - 25\%

4. Nicoline Potgieter: Scientific writing and proofreading of the manuscript - $25 \%$
In a study by Holcomb and Gregory, ${ }^{15} 881$ participants were investigated for the presence of $\mathrm{CM}$. Initial periapical radiographs were taken of the participant and repeated four years later.

The survey revealed an incidence of partial or complete canal obliteration of $3.86 \%$ with initial examination. Only three $(7.3 \%)$ of the 41 teeth developed periradicular rarefactions after the four-year follow-up. Over $79 \%$ of the participants could recall a traumatic dental injury between the age of 10 and 16 years of age. The authors of this study concluded that a significant correlation exists between a prior traumatic dental injury and the incidence of CM. They also commented that associated periapical rarefaction is uncommon and that endodontic intervention should only be considered in rare circumstances.

In a follow-up study by Andreasen, ${ }^{6}$ where 189 teeth with history of dental luxation were examined, the authors described $\mathrm{CM}$ as an accelerated deposition of dentine and did not advocate early endodontic intervention. Pulp canal obliteration was observed in $22 \%$ of luxated teeth over a mean observation period of 3.4 years. Only $7 \%$ of teeth became necrotic over the follow up time. A relationship was found between the incidence of pulp obliteration and the stage of root development. The incidence of $\mathrm{CM}$ was higher in teeth with incomplete root development.

Another study by Andreason et al., ${ }^{18}$ showed a $5 \%$ incidence of CM in 637 permanent incisors after luxation injuries. Only $1 \%$ of these teeth developed pulpal necrosis when evaluated over a five-year period.

Stalhane and Hedegard ${ }^{19}$ conducted a long-term study on 76 teeth that showed $\mathrm{CM}$ following traumatic injuries. Follow-up examinations were done 3-21 years after injuries. Of the 76 teeth, 12 teeth (16\%) developed periapical rarefaction over this examination period.

The stage of root development, time of trauma as well as type of traumatic injury were also recorded. The authors commented on the fact that the success of modern endodontic treatment should be weighed against the incidence of developing periapical pathology when deciding on clinical treatment options.

A study by Jacobsen and Kerekes, ${ }^{20}$ followed up on radiographic hard tissue changes of 122 teeth, 10-23 years post injury. Of the $36 \%$ of cases with partial and $64 \%$ of cases with complete canal obliteration, none of the partially obliterated canals became necrotic with 
peri-apical rarefactions and only $13 \%$ of the complete obliterations eventually developed pulpal necrosis. The findings of this study strengthen the rationale behind performing endodontic treatment only when periradicular rarefactions develop.

In a study by Robertson et al., ${ }^{21} 82$ permanent incisors with a history of trauma were followed up over a period of 7-22 years. Frequent yellow discolouration was seen and by evaluating periradicular bone loss, the authors reported that $8.5 \%$ of cases developed pulpal necrosis over this time.

The survival rate was found to be $84 \%$ after 20-years and it was reported that caries, new trauma, orthodontic treatment as well as crown coverage did not increase the frequency of pulpal necrosis.

Rock and Grundy ${ }^{7}$ suggested a different approach to the previous studies and did a retrospective study in which 517 traumatised teeth were evaluated for the development of CM. They found that $16 \%$ of teeth developed $\mathrm{CM}$. Although not statistically significant, CM was seen in the younger age groups (<9 years) while root resorption was seen in the older age group ( $>9$ years).

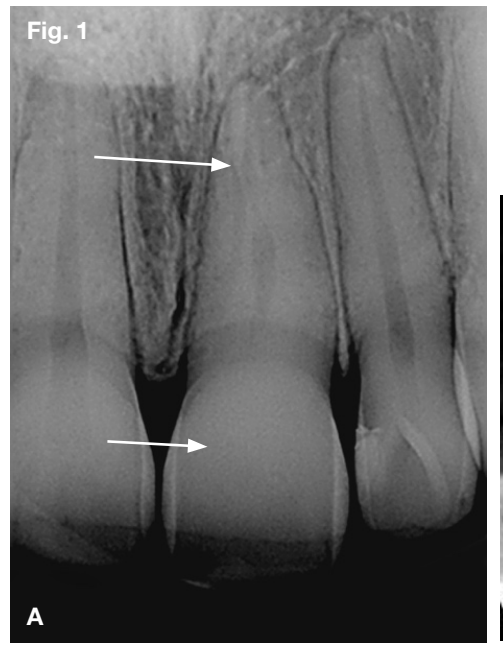

Figure 1.

A. Periapical radiograph of a left maxillary $B$. central incisor of a 27-year-old female patient that suffered a concussion injury at the age of 14 years. Note the canal obliteration in the coronal and apical third of the root canal (arrows), leaving a small amount of pulp tissue in the mid-root region.

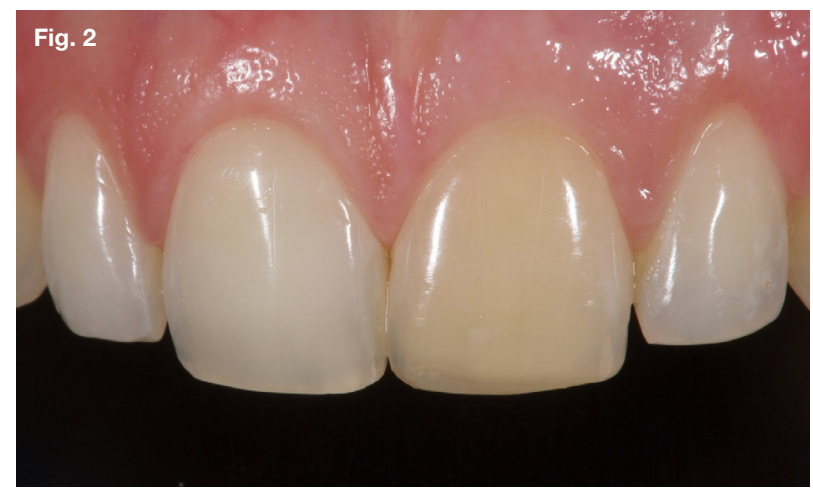

Figure 2. Clinical view of the discoloured left maxillary central incisor. illary arch.

\section{Clinical, radiographic and histological findings}

Most teeth presenting with canal obliteration are asymptomatic ${ }^{1,9,21}$ including the absence of sensitivity to percussion. ${ }^{10} \mathrm{CM}$ is therefore often an incidental finding during clinical or radiographic investigations., ${ }^{1,9}$

$\mathrm{CM}$ is usually characterized by a dark yellow discolouration of the tooth..$^{9,10}$ This distinct discolouration of the crown has been reported in $79 \%$ of 122 teeth with pulpal obliteration $^{9,20}$ and may be attributed to the deposition of dark tertiary dentine..$^{8,10,13,14}$

Oginni et al. ${ }^{1}$ reported that out of 276 teeth with partial canal obliteration, 186 (67\%) had yellow discoloration and a further 34 (12\%) teeth were grey in colour.

Interestingly, the incidence of pulp or periapical pathology were greater in the teeth with a grey discoloration compared to those with yellow discoloration. ${ }^{1}$ It is important to note that teeth with radiographic signs of pulpal obliteration may also present without any colour change, ${ }^{9}$ and although colour change may indicate $\mathrm{CM}$, it is not a necessarily an indication of pulpal or periapical pathosis. ${ }^{1,9,20,21}$

In the presence of partial canal obliteration, it is generally accepted that vitality tests are unreliable.1,9,21,22 These teeth show a delayed or completely absent response to vitality tests. ${ }^{10,11,23}$ The response to vitality tests also tend to decrease as pulpal obliteration progresses. ${ }^{1,5,9,16}$
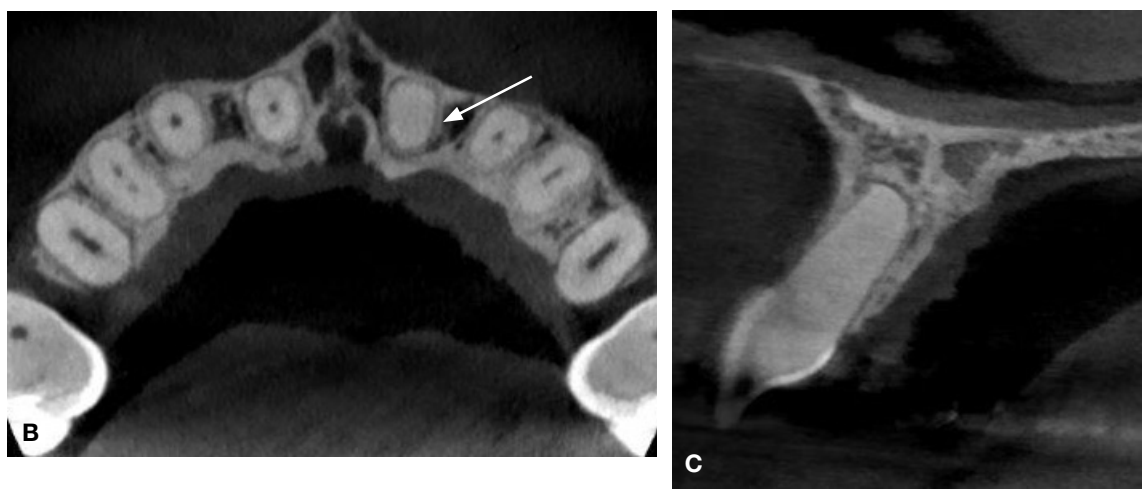

Axial slice of CBCT at the level of CEJ showing COM- $C$. Sagittal slice of CBCT showing complete canal obliteration of the left maxillary central plete canal obliteration of the left maxincisor (arrow) compared to the other teeth in max- illary central incisor.

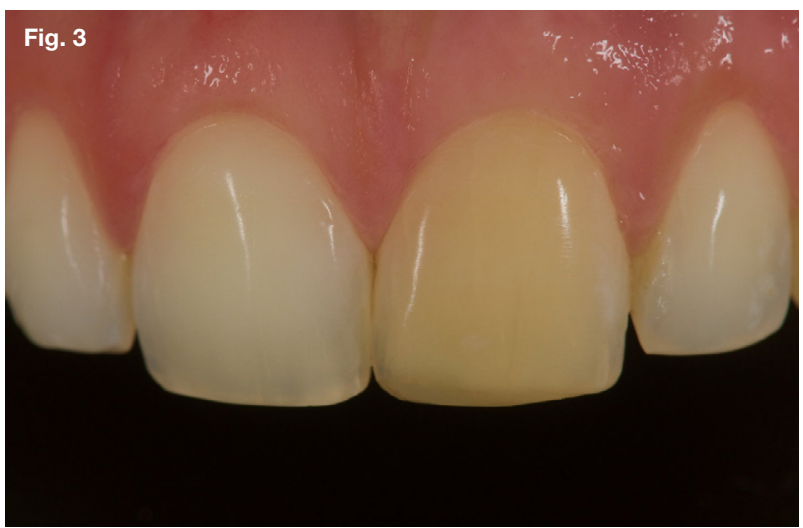

Figure 3. Clinical view of the discoloured left maxillary central incisor taken with cross polarization photography resulting in no specular reflection. Note the severe change in hue and value of the affected tooth. 
Generally, canal obliteration progresses in a corono-apical direction. ${ }^{10} \mathrm{~A}$ decrease in the pulp chamber volume is often the first radiographic sign, followed by a gradual narrowing of the complete root canal. ${ }^{10,23}$

Obliteration can be classified as either partial (coronal), or complete (extending radicular) obliteration of the pulp canal space. ${ }^{9,10} \mathrm{CM}$ usually presents with a normal periodontal ligament space and intact lamina dura. ${ }^{11,14,23,24}$

Thickening of the periodontal ligament space or periradicula pathology may however be visible in the presence of infection. $9,10,14,24$

Cone beam Computed Tomography (CBCT) may prove helpful in locating the root canals. ${ }^{10,24-26}$ The option to view canals in multiplanar reconstructions provides the clinician with valuable information in the diagnosis and management of CM. ${ }^{10,27}$

The absence of a root canal on conventional radiographs does not necessarily mean total absence of that canal. $5,8,9,16$ Histologic evaluation of pulp canals, radiographically diagnosed as being obliterated, almost always confirm the existence of a narrow pulp canal containing pulpal tissue. ${ }^{10,12,14,23}$ Canal mineralization has therefore been suggested as a more accurate term as opposed to canal obliteration. ${ }^{10,23,28}$

Pulp canal obliteration was initially described as an irregular tertiary dentine deposition, but more recently as multifocal, dystrophic calcifications composed of illdefined secondary dentine.,20-31 Calcifications have also been described by some authors as dentine-like, bonelike and fibrotic. ${ }^{10,32,33}$ Holan $^{24}$ described calcificationsas tube-like osteo-dentine structures extending along the entire length of the pulp canal, surrounded by pulp tissue only connected to the root dentine at some areas.

In a study by Lundberg and Cvek, ${ }^{32}$ no micro-organisms were found in any of the specimens investigated. Inflammatory components, indicative of a pathologic process, are usually absent in teeth with CM. $5,8,10,13,4,32,34$ Based on these histopathological studies, asymptomatic $\mathrm{CM}$ does not indicate the necessity for root canal treatment. ${ }^{10,14,18,32}$

\section{Treatment options}

The dark appearance of teeth affected by CM is not only a huge aesthetic concern but also presents the clinician with a great treatment challenge. Recently, West ${ }^{35}$ suggested four potential treatment options for the restoration of aesthetics in discoloured teeth affected by sclerosis of the root canal complex. The four treatment options with an example of a case report on option one and two are described below.

\section{Vital bleaching/External bleaching}

Due to its conservative nature, several authors advocate that external or vital bleaching should be considered as a first treatment option. ${ }^{35-38}$ Greenwall $^{37}$ described a vital tooth whitening technique for affected single teeth. The author advocated the use of $20 \%$ carbamide pe- roxide gel in a modified conventional vital bleaching tray where windows are created adjacent to the discoloured tooth, on either side.

This modification will prevent the overflow of bleaching liquid to adjacent teeth which might result in uneven whitening. The disadvantage of this particular technique is that progress can be slow due to the nature of discoloration and the final result might not be acceptable. An advantage of this treatment option is that patients experience limited or no sensitivity during the whitening procedure. $^{37}$

Joiner ${ }^{39}$ suggested that using a low con-centration for a longer treatment time will reduce the negative effects (as a result of rapid diffusion of free radicals) of bleaching. Additionally, the prolonged use of bleaching products with a low concentration (for example 10-20\% carbamide peroxide) provides greater stability. ${ }^{39}$

Haywood and DiAngelis ${ }^{40}$ discussed two approaches when considering external bleaching as a treatment option to improve aesthetics:

Tray bleaching: the authors advocated a horseshoeshaped cast on which the tray is manufactured with no vestibule to allow proper adaptation of bleaching solution.

Single tooth bleaching tray: a non-scalloped, non-reservoir tray modified and trimmed to fit a single discoloured tooth. Patients are provided with a single syringe bleaching solution.

\section{CASE REPORT}

A 29-year-old female presented with a history of trauma to her maxillary anterior teeth at the age of 13 and agenesis of her maxillary left and right lateral incisors (Fig. 4). Historically, orthodontic treatment was attempted to move the canines into the position of the laterals. Following orthodontic treatment the patient relapsed with a resultant non-aesthetic appearance.

The main concern was a yellow discoloration of both the maxillary canines and left central incisor (Fig. 4). The patient sought an immediate aesthetic solution as she was getting married in four weeks' time. Radiographic examination revealed complete canal obliteration of the asymptomatic maxillary left central incisor (Fig. 5A), with vital and visible root canal systems on the canines (Fig. 5B).

It was decided to manage the case using the vital bleaching technique with 16\% carbamide peroxide (Flash Take Home Whitening System, Whitesmile GmbH, Germany) for three weeks. The patient was instructed to bleach the discoloured teeth every day for 45 minutes and the rest of the arch was bleached every third day.

Figure 6 shows the result after vital bleaching and Figure 7 the immediate postoperative result after minimally invasive direct composite restorations to modify the anatomical shape of the teeth and to close the diastema between the 21 and 23 . 


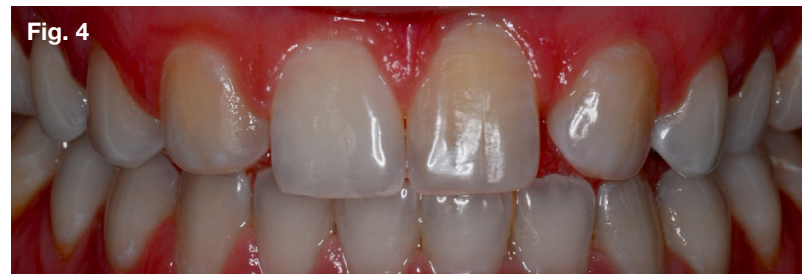

Figure 4. Yellow discoloration of the left central incisor and both maxillary canines, in the position of the laterals.
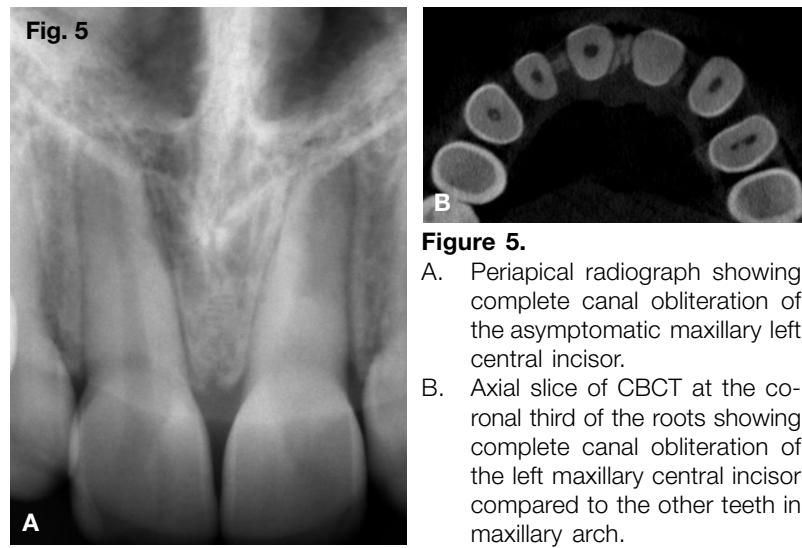

Figure 5.

A. Periapical radiograph showing complete canal obliteration of the asymptomatic maxillary left central incisor.

B. Axial slice of CBCT at the coronal third of the roots showing complete canal obliteration of the left maxillary central incisor compared to the other teeth in maxillary arch.

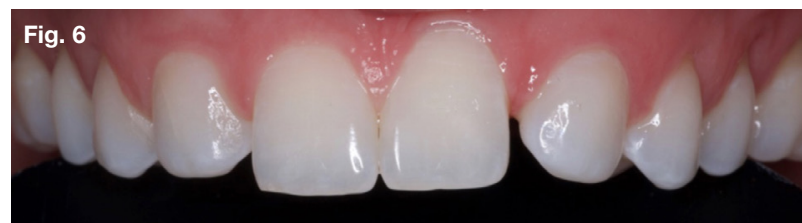

Figure 6. Postoperative result after vital bleaching

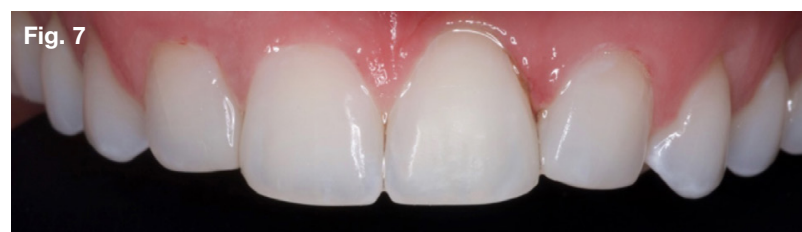

Figure 7. Postoperative result after direct composite restorations on the left central incisor and two canines.

\section{Intentional root canal treatment followed by intra-coronal/non-vital bleaching}

Rock and Grundy ${ }^{7}$ recommended root canal treatment as soon as evidence of narrowing of the pulp chamber was seen radiographically.

The rationale was based on two clinical considerations: firstly, once access to the canal is lost there is an increased risk of root perforation and secondly, a loss of coronal access to the root canal might result in surgical intervention should the remainder of the canal become necrotic.

De Cleen $^{11}$ recommended a more invasive approach by creating a fully extended access cavity similar to a healthy unaffected tooth with normal chamber size.

The author was convinced that by following this approach, the majority of tertiary dentine will be removed and ultimately create a translucency within the crown.

Rotstein \& Walton ${ }^{41}$ concluded that an acceptable aesthetic result could be obtained on $\mathrm{CM}$ affected teeth by means of intra-coronal bleaching, once root canal treatment has been completed successfully. However, the disadvantage of the non-vital bleaching technique is the increased possibility of colour regression over time.

The exact mechanism responsible for this colour regression is not yet defined although micro leakage through the final covering restoration could play a role. ${ }^{42}$

Friedman et al. ${ }^{43}$ observed endodontically treated teeth with non-vital bleaching and found that on recall periods of one to eight years, $79 \%$ of these teeth illustrated improved colour and aesthetics compared to their initial appearance. It should however be stated that the introduction of bleaching solution into the pulp chamber may increase the risk of external cervical resorption. ${ }^{44}$ Bleaching procedure for endodontically treated anterior teeth can be approached internally, externally or a combination of both depending on a proper assessment and diagnosis. Haywood and DiAngelis ${ }^{40}$ discussed different approaches when considering bleaching as a treatment option in non-vital teeth:

In-office bleaching: the classic in-office non-vital bleaching technique involves the use of a high concentration hydrogen peroxide (35\%) which is placed in the prepared pulp chamber and activated with a light or heat source.

Walking bleach technique: a classic technique where gutta-percha is removed $2 \mathrm{~mm}$ below the cemento-enamel junction, sealed off using a lining material (such as glass ionomer) with bleaching solution placed and sealed in. The patient is instructed to return for subsequent visits and follow-ups.

Internal-external closed bleaching: bleaching is executed from within the affected tooth as well as the external surface. The pulp chamber is prepared and the walking bleach technique is used as described above. Additionally, a single tooth tray is manufactured to allow bleaching externally and patient follows the instructions as advocated above.

According to Haywood and DiAngelis and other authors, this approach is considered as most effective and proves most beneficial. ${ }^{40,45,46}$

Internal-external open bleaching: the technique is similar to the internal-external closed technique accept that the affected tooth is left open and the patient is instructed to fill the tooth and tray with bleaching solution. The technique is highly reliant on the patient's understanding and co-operation.

For all approaches it is crucial to remove any remnants of pulp horns before the internal bleaching procedure is conducted. Very often proper cleaning of the pulp chamber and pulp horns will result in an improved appearance.

\section{CASE REPORT}

A 27-year-old female presented with percussion sensitivity on her maxillary left central incisor. She was also concerned about a yellow-brown discoloration of this 
tooth (Fig. 8) and expressed the desire to have her teeth "bleached". A peri-apical radiograph and CBCT scan revealed that the canal was almost completely obliterated (Fig. 9).

The tooth was isolated with rubber dam using a Brinker Tissue Retractor (Hygenic) clamp (Fig. 10) to ensure retention of the clamp because of the lack of an adequate cingulum area on the palatal aspect of the tooth.

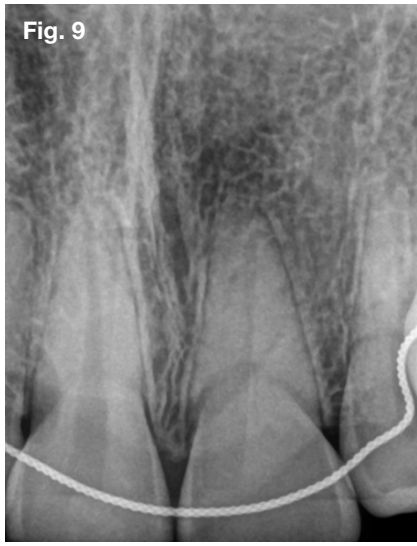

Figure 9. Pre-operative peri-apical radiograph of maxillary left central incisor with obliterated root canal system.
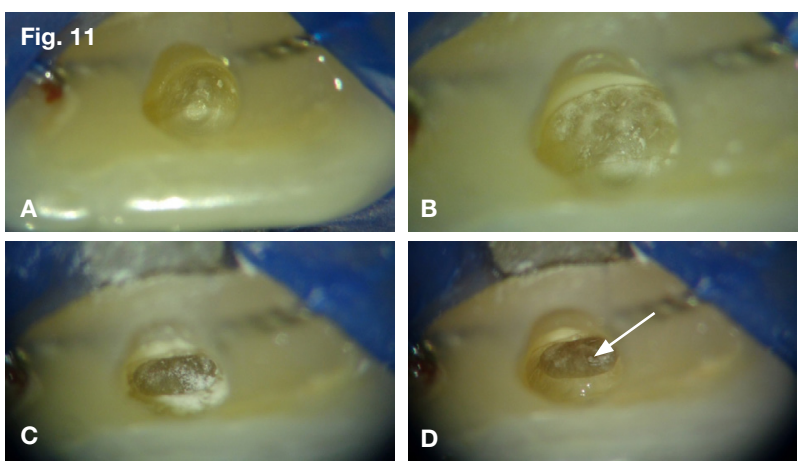

Figure 11. High magnification views of the pulp chamber floor.

A. Initial view after access cavity preparation. Note the darker yellow discoloration outlining the position of the original pulp chamber.

B. Selective removal of the darker dentine with a small long-shank bur (Dentsply Sirona). Clearly visible is the outline of the tertiary dentine formation and calcification of the original root canal system.

C. Troughing to a deeper level revealed narrow band of darker discoloured dentine directly related to the smaller diameter of the original root canal system at this level.

D. After troughing to a deeper level with a Start.X ultrasonic tip (Dentsply Sirona) a narrow band of reactionary dentine was revealed with a collection of white dentine debris in one spot (arrow), indicating the remaining canal space.

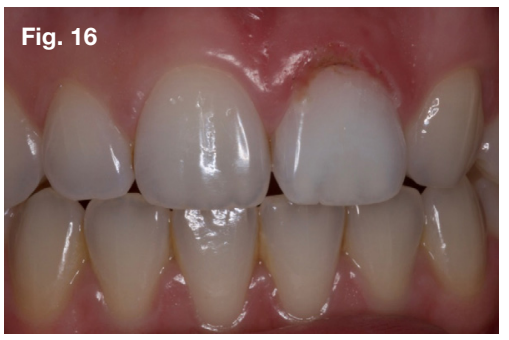

Figure 16. Result after bleaching technique for 48 hours using 35\% hydrogen peroxide until a lighter value was obtained and the uneven gingival contour of the tooth was corrected using a Sirolase Blue (Dentsply Sirona).

After access cavity preparation, the darker dentine discoloration of the pulp floor was followed with small longshank burs (Dentsply Sirona) and a Start.X ultrasonic tip (Dentsply Sirona) until a rather calcified canal orifice was located (Fig. 11A-D). The coronal aspect of the canal was negotiated with a size $08 \mathrm{C}+$ file (Dentsply Sirona) (Fig. 12) followed by a $08 \mathrm{~K}$-file. This sequence was repeated until canal patency and full working length (Fig. 13) were achieved.

A size $10 \mathrm{~K}$-file was used to create a reproducible micro glide path before the macro glide path was completed using the TruNatomy Glider (Dentsply Sirona) in 8-12 back-stroke brushing motions. Canal preparation was done with the TruNatomy Prime (Dentsply Sirona) file followed by canal irrigation with 17\% EDTA (Vista Dental) and 3.5\% sodium hypochlorite.
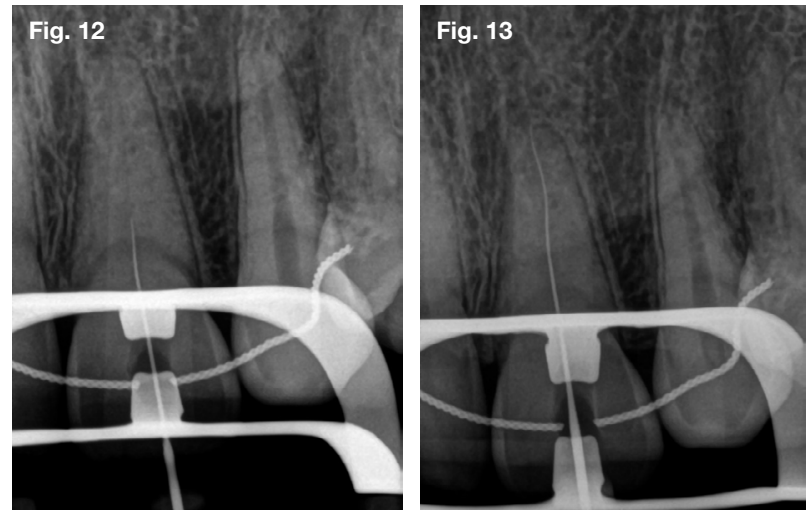

Figure 12. Coronal aspect of the root canal negotiated with a $08 \mathrm{C}+$ file

Figure 13. Length determination on peri-apical radiograph.

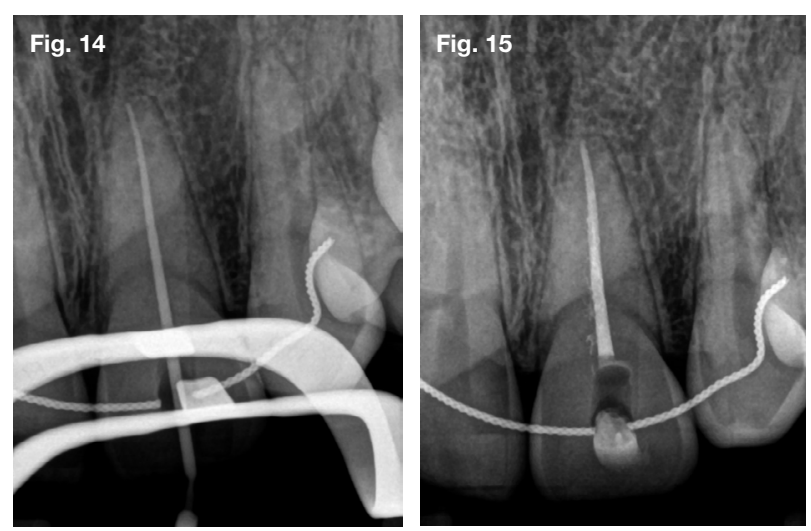

Figure 14. Conefit peri-apical radiograph confirming the fit of a TruNatomy Prime Gutta Percha Point (Dentsply Sirona).

Figure 15. Postoperative peri-apical radiograph after root canal obturation.

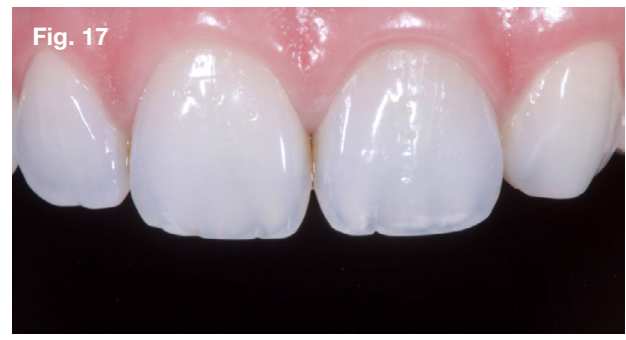

Figure 17. Final aesthetic result that was obtained after the treatment.

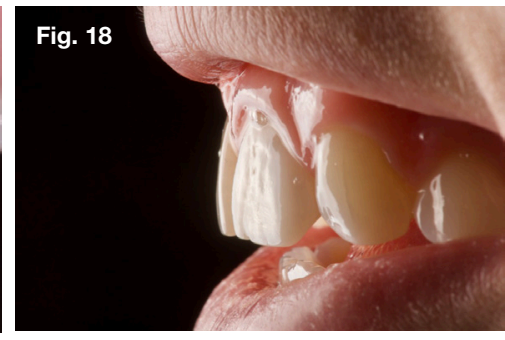

Figure 18. Side view illustrating the aesthetic outcome of the treatment resulting in a very happy patient. 
A TruNatomy Prime Gutta Percha Point (Dentsply Sirona) was placed in the prepared root canal system and the fit verified radiographically (Fig. 14). Finally, the root canal system was obturated with a TruNatomy Prime Gutta Percha Point, AH Plus sealer (Dentsply Sirona) and the Gutta Smart Obturation System (Dentsply Sirona) (Fig. 15).

The tooth was bleached utilising the walking bleach technique for 48 hours using 35\% hydrogen peroxide (Opal Endo, Ultradent) until a lighter value was obtained compared to the other maxillary teeth. The uneven gingival contour of the tooth was corrected using a Sirolase Blue (Dentsply Sirona) (Fig. 16).

This was followed by home bleaching of the other maxillary teeth using 10\% hydrogen peroxide (Flash Take Home Whitening System). The palatal access cavity was cleaned with air polishing before it was restored with SDR (Dentsply Sirona) and composite resin.

Figure 17 and 18 shows the final aesthetic result that was obtained after the treatment.

\section{Internal and external bleaching without root canal treatment}

Pedorella, Meyer and Woollard ${ }^{47}$ described a technique where the access cavity is prepared by removing the sclerotic dentine in the coronal portion of the affected tooth followed by the placement of a suitable base/liner on the floor of the prepared cavity. The approach is aimed at addressing the aesthetic concerns with internal and external bleaching without attempting the root canal procedure.

Whilst considering this approach as a suitable treatment option, the technique is not well supported in literature and does not have widespread support. Various studies have concluded that in the majority of cases a pulp space with pulp tissue is present.

Conventional radiographs fail to capture the presence of a microscopic root canal system, therefore being interpreted as a completely sclerotic tooth with no root canal and pulp chamber. ${ }^{5,8,16}$ Once the cavity preparation for intra-coronal bleaching is attempted and a microscopic root canal system in unknowingly penetrated, the pulp tissue is exposed to possible infection that might result in periradicular pathology. ${ }^{9}$

\section{Extra-coronal full or partial coverage}

Traditionally, various invasive treatment approaches were followed in an effort to improve the appearance of teeth affected by CM. Direct or indirect veneers, ceramic crowns or removal of part of the palatal dentine (similar to traditional endodontic access) are among the treatment approaches attempted to restore aesthetics.

The disadvantage of these invasive approaches is the removal of healthy tooth structure and weakening of the remaining tooth structure. ${ }^{44}$ Recent reports have suggested external bleaching as the first treatment option. This treatment is non-invasive, relatively cost effective and simple with predictable outcomes. ${ }^{48}$
West ${ }^{35}$ suggested a more invasive treatment option by considering a full coverage restoration. Considering the fact that most teeth affected by $\mathrm{CM}$ are intact and have no structural damage, the preparation and placement of a full or partial covering restoration should be carefully considered only when more conservative approaches have failed. Malhotra ${ }^{10}$ also suggested the preparation and placement of veneers on teeth with large existing restorations, fractures or defects. A combination of treatment options can also be considered.

\section{CONCLUSION}

Treatment of CM, both endodontically and aesthetically, remain challenging for even the skilled clinician. In this paper the authors provide an overview of $\mathrm{CM}$ and some clinical advice on the management this condition both endodontically and aesthetically. An accurate diagnosis, based on clinical and radiographic findings, will guide the practitioner to select the appropriate treatment approach or combination of approaches for each individualised case.

\section{Declaration}

The authors have no conflict of interest to declare.

\section{Clinical significance}

This review article aims to give the reader an in depth oversight on the aetiology and clinical presentation of calcific metamorphosis. The authors also provide some insight on the clinical management of these, often complexed and difficult to manage clinically, cases.

\section{References}

1. Oginni AO, Adekoya-Sofowora CA, Kolawole KA. Evaluation of radiographs, clinical signs and symptoms associated with pulp canal obliteration: an aid to treatment decision. Dent Traumatol. 2009; 25(6): 620-5.

2. Andreasen JO, Andreasen FM, Andersson L. Textbook and color atlas of traumatic injuries to the teeth. 4th ed. Copenhagen: Munksgaard International Publishers. 2007; 411-27.

3. Yaacob H, Hamid JA. Pulpal calcifications in primary teeth: a light microscope study. J Pedod. 1985; 10(3): 254-64.

4. Robertson A. A retrospective evaluation of patients with uncomplicated crown fractures and luxation injuries. Dent Traumatol. 1998; 14(6): 245-56.

5. Patterson SS, Mitchell DF. Calcific metamorphosis of the dental pulp. Oral Surg Oral Med Oral Pathol. 1965; 20(1): 94-101.

6. Andreasen J. Luxation of permanent teeth due to trauma. A clinical and radiographic follow-up study of 189 injured teeth. Eur J Oral Sci. 1970; 78(1-4): 273-86.

7. Rock W, Grundy M. The effect of luxation and subluxation upon the prognosis of traumatized incisor teeth. $J$ Dent. 1981; 9(3): 224-30.

8. Torneck C. The clinical significance and management of calcific pulp obliteration. The Alpha omegan. 1990; 83(4): 50-4.

9. McCabe P, Dummer PMH. Pulp canal obliteration: an endodontic diagnosis and treatment challenge. Int Endod J. 2012; 45(2): 177-97. 
10. Malhotra N, Mala K. Calcific metamorphosis. Literature review and clinical strategies. Dent Update. 2013; 40(1): 48-60.

11. de Cleen M. Obliteration of pulp canal space after concussion and subluxation: endodontic considerations. Quintessence Int. 2002; 33(9): 661-69.

12. Feiglin B. Dental pulp response to traumatic injuries-a retrospective analysis with case reports. Dent Traumatol. 1996; 12(1): 1-8.

13. Gopikrishna V, Parameswaran A, Kandaswamy D. Criteria for management of calcific metamorphosis: review with a case report. Indian J Dent Res. 2004; 15(2): 54-7.

14. Amir FA, Gutmann JL, E Witherspoon D. Calcific metamorphosis: a challenge in endodontic diagnosis and treatment. Quintessence Int. 2001; 32(6): 447-55.

15. Holcomb JB, Gregory WB. Calcific metamorphosis of the pulp: its incidence and treatment. Oral Surg Oral Med Oral Pathol. 1967; 24(6): 825-30.

16. Schindler WG, Gullickson DC. Rationale for the management of calcific metamorphosis secondary to traumatic injuries. J Endod. 1988; 14(8): 408-12.

17. Smith JW. Calcific metamorphosis: a treatment dilemma. Oral Surg Oral Med Oral Pathol. 1982; 54(4): 441-4.

18. Andreasen FM, Zhjie Y, Thomsen BL, Andersen PK. Occurrence of pulp canal obliteration after luxation injuries in the permanent dentition. Dent Traumatol. 1987; 3(3): 103-15.

19. Stalhanse I. Traumatized permanent teeth in children aged 715 years, Part II. Swed Dent J. 1975; 68: 157-69.

20. Jacobsen I, Kerekes K. Long-term prognosis of traumatized permanent anterior teeth showing calcifying processes in the pulp cavity. Eur J Oral Sci. 1977; 85(7): 588-98.

21. Robertson A, Andreasen FM, Bergenholtz G, Andreasen JO, Norén JG. Incidence of pulp necrosis subsequent to pulp canal obliteration from trauma of permanent incisors. J Endod. 1996; 22(10): 557-60.

22. Holcomb JB, Gregory WB. Calcific metamorphosis of the pulp: its incidence and treatment. Oral Surg Oral Med Oral Pathol. 1967; 24(6): 825-30.

23. Abbott $P$, Yu C. A clinical classification of the status of the pulp and the root canal system. Aust Dent J. 2007; 52(1): 17-31.

24. Holan G. Tube-like mineralization in the dental pulp of traumatized primary incisors. Dent Traumatol. 1998; 14(6): 279-84.

25. Tyndall DA, Rathore S. Cone-beam CT diagnostic applications: caries, periodontal bone assessment, and endodontic applications. Dent Clin N Am. 2008; 52(4): 825-41.

26. Mora MA, Mol A, Tyndall DA, Rivera EM. In vitro assessment of local computed tomography for the detection of longitudinal tooth fractures. Oral Surg Oral Med Oral Pathol Oral Radiol Endod. 2007; 103(6): 825-9.

27. Nair MK, Nair UP. Digital and advanced imaging in endodontics: a review. J Endod. 2007; 33(1): 1-6.

28. Levin LG, Law AS, Holland G, Abbott PV, Roda RS. Identify and define all diagnostic terms for pulpal health and disease states. J Endod. 2009; 35(12): 1645-57.

29. Johnson PL, Bevelander G. Histogenesis and histochemistry of pulpal calcification. J Dent Res. 1956; 35(5): 714-22.

30. Eversole LR. Clinical outline of oral pathology: diagnosis and treatment. Lea and Febiger, Philadelphia: 1978; 273-4.

31. Kuster CG. Calcific metamorphosis/internal resorption: a case report. Pediatr Dent. 1981; 3(3): 274-5.

32. Lundberg M, Cvek M. A light microscopy study of pulps from traumatized permanent incisors with reduced pulpal lumen. Acta Odontol Scand. 1980; 38(2): 89-94.
33. Oginni AO, Adekoya-Sofowora CA. Pulpal sequelae after trauma to anterior teeth among adult Nigerian dental patients. BMC Oral Health. 2007; 7(1): 1-5.

34. Cvek M, Granath L, Lundberg M. Failures and healing in endodontically treated non-vital anterior teeth with posttraumatically reduced pulpal lumen. Acta Odontol Scand. 1982; 40(4): 223-8.

35. West J. The aesthetic and endodontic dilemmas of calcific metamorphosis. Pract Periodontics Aesthet Dent. 1997; 9(3): 289-93.

36. Munley P, Goodell G. Calcific metamorphosis. Clin Update. 2005; 27(4).

37. Greenwall L. Single vital tooth whitening. Int Dent SA. 2007; 10(1): 48-52.

38. Borkar N, Jaggi S, Pandit V, Shetty S. Calcific Metamorphosisa review. IIOABJ. 2016; 7(6): 71-6.

39. Joiner A. The bleaching of teeth: a review of the literature. J Dent. 2006; 34(7): 412-9.

40. Haywood VB, DiAngelis AJ. Bleaching the single dark tooth. Inside Dent. 2010; 6(8): 42-52.

41. McDonald NJ, Torabinejad M. Endodontic surgery. Principles Practice Endod. 2002; 3: 357-75.

42. Friedman S. Internal bleaching: long-term outcomes and complications. J Am Dent Assoc. 1997; 128: 51-5.

43. Friedman S, Rotstein I, Libfeld H, Stabholz A, Heling I. Incidence of external root resorption and esthetic results in 58 bleached pulpless teeth. Dent Traumatol. 1988; 4(1): 23-6.

44. Ramos TM, Oliveira TMR, de Azevedo CS, de Gois DN, de Aguiar Oliveira AH, de Freitas PM. Conservative esthetic treatment of a discoloured calcified permanent tooth: five-year clinical evaluation. Braz Dent Sci. 2013; 16(4): 105-12.

45. Deliperi S, Bardwell DN, Papathanasiou A. Clinical evaluation of a combined in-office and take-home bleaching system. J Am Dent Assoc. 2004; 135(5): 628-34.

46. Delgado E, Hernández-Cott PL, Stewart B, Collins M, de Vizio W. Tooth-whitening efficacy of custom tray-delivered $9 \%$ hydrogen peroxide and 20\% carbamide peroxide during daytime use: a 14-day clinical trial. Puerto Rico Health Sci J. 2009; 26(4): 367-72.

47. Pedorella C, Meyer R, Woollard G. Whitening of endodontically untreated calcified anterior teeth. Gen Dent. 2000; 48(3): 252-5.

48. Kwon SR. Whitening the single discolored tooth. Dent Clin N Am. 2011; 55(2): 229-39. 